\title{
MedienPädagogik
}

Zeitschrift für Theorie und Praxis der Medienbildung www.medienpaed.com

Themenheft Nr. 25: Medienbildung und informatische Bildung - quo vadis? Hrsg. von Klaus Rummler, Beat Döbeli Honegger, Heinz Moser und Horst Niesyto

\section{Medienbildung und Informatische Bildung - Interdisziplinäre Spurensuche}

Bardo Herzig

\section{Zusammenfassung}

Die Diskussion um das Verhältnis von Medienbildung und Informatischer Bildung', so die Argumentation im vorliegenden Beitrag, sollte von einem umfassenden Medienbegriff ausgehen, der bereits auf die Spezifika Digitaler Medien, insbesondere ihre technische Bedingtheit, hinweist. Zeichnet man den intradisziplinären Diskurs nach, so zeigt sich, dass die Medienpädagogik den Computer als ein neues Medium in der Entwicklung der Medienlandschaft quasi selbstverständlich in ihren disziplinären Gegenstand integriert hat, die Thematisierung informatischer Aspekte aber eher die Ausnahme bildet. Erst ein Blick auf stärker medienwissenschaftlich und informatisch ausgerichtete Arbeiten legt interdisziplinäre Aspekte offen, die von der Maschinenebene bis hin zur Interaktion mit Digitalen Medien eine durchgängige Beschreibung von Strukturen und Prozessen erlauben. Solche Aspekte repräsentieren zentrale bzw. fundamentale Ideen und Prinzipien, wie z. B. Semiotisierung, Formalisierung, Software oder Interaktivität. Die Frage, wie solche Ideen in den Bildungsdiskurs Eingang finden, sollte an die Debatte um Medienkompetenzmodelle und Standards anknüpfen. Die unterrichtliche Umsetzung einer umfassenden Medienbildung ist - vor dem Hintergrund der zentralen interdisziplinären Aspekte - auf eine explizite informatische Expertise angewiesen.

\section{Media education and informatics education - an interdisciplinary search for traces}

\begin{abstract}
The discussion about the relation between media education and informatics education should - so the argumentation in this article - start from a comprehensive media term, which already emphasizes the specific features of digital media. Portraying the intradisciplinary discourse it becomes apparent, that media education as a discipline has integrated the computer as a new medium as a matter of course, but that the thematization of aspects of informatics is rather the exceptional case. Just viewing such approaches being influenced by media studies or informatics reveal interdisciplinary

1 〈Informatische Bildung〉 und «Digitale Medien` werden als eigenständige Begriffe verstanden und gross geschrieben.
\end{abstract}


aspects allowing a consistent description and illumination of structures and processes from the machine level through to the level of interaction with digital media. Such aspects represent fundamental ideas and principles like semiotization, formalization, software or interactivity. The question how interdisciplinary ideas find its way into the educational discourse should affiliate to the debate about models of media competences and standards. The instructional and educational implementation of a comprehensive media education depends - with regard to the central interdisciplinary aspects - on an explicit expertise in informatics.

\section{Einführung}

Angesichts der Vielzahl von Begrifflichkeiten und Konzepten in der Diskussion um 〈Informatische Bildung〉 und 'Medienbildung〉 erweist sich der Versuch, etwas zu diesem Themenbereich beizutragen, schon aus semantischen Gründen als schwierig. So könnte der Beitrag auch mit Medienkompetenz, Digitaler Kompetenz, Informatischer Kompetenz oder Digitaler Bildung beginnen, ohne dass dies schon Hinweise auf mögliche Argumentationslinien liefern könnte. Dass dieser Diskurs rege ist, sei nur angedeutet: So stehen Vorschläge, von einer informatischen Bildung auszugehen, die auch Medienbildung mit beinhaltet (vgl. z. B. Döbeli Honegger 2013a; 2013b; Kleiner 2014) neben dem Plädoyer für ein ssowohl als auch» anstatt eines «entweder - oder) (vgl. Spannagel 2015a; 2015b) oder einer Zusammenführung von Informatik und Medienbildung in einem gemeinsamen Fach (vgl. Reiter 2010). Zuweilen wird aber auch die Medienbildung zugunsten eines Kampfes gegen den informatischen Analphabetismus als überflüssig oder lächerlich abgetan (vgl. Hromkovic 2012). Insgesamt geht es nicht nur um inhaltliche Aspekte, sondern auch um disziplinäre Aspekte. Subsumptionen des einen Bereichs unter den anderen, aber auch integrative Lösungen werden mit der Gefahr der Trivialisierung der Informatik oder auch der Medienbildung verbunden (vgl. z. B. Döbeli Honegger 2013b, Moser 2013; Spannagel 2015b).

Das Gebiet ist also ein Stück weit vermint, gleichwohl aber noch nicht zufriedenstellend vermessen. Im vorliegenden Beitrag geht es um die Frage, wie das Interdisziplinäre zwischen Informatik und Medienpädagogik bestimmt und in den Bildungsdiskurs eingebracht werden kann. Eine interdisziplinäre Diskussion sucht nach dem Raum zwischen den Disziplinen, was impliziert, dass dabei die Eigenständigkeit der Disziplinen nicht in Frage gestellt wird, sondern nach Schnittmengen oder mindestens nach Anschlussstellen gefragt wird. Im vorliegenden Beitrag wird zunächst ein breiter, interdisziplinär anschlussfähiger Medienbegriff skizziert, bevor danach gefragt wird, welches (Selbst-)Verständnis die Medienpädagogik und die Informatik als Theorien der Erziehungs- und Bildungspraxis bzw. der maschinellen Informationsverarbeitung in Bezug auf die Rolle des Computers als Medium 
entwickelt haben. In einem weiteren Schritt wird dann - auf einer Metaebene nach beiden Wissenschaftsbereichen zugrunde liegenden Verbindungen gesucht. Von solchen Grundlagen aus kann dann die Frage nach ableitbaren interdisziplinären Bildungs- oder Erziehungszielen und ihrer Umsetzung angegangen werden.

\section{Interdisziplinärer Medienbegriff}

Medienpädagogik und Informatik - als die Bezugsdisziplinen für Medien- bzw. Informatische Bildung - beziehen sich auf ein gemeinsames Artefakt, den Computer. Während es für die Informatik das zentrale Artefakt ist (was nicht bedeutet, dass sich die Informatik nur mit Computern beschäftigt), stellt der Computer in der Medienpädagogik eines von vielen Artefakten dar, das unter die Palette von Medien subsumiert werden könnte. Gerade eine solche quasi selbstverständliche begriffliche Vereinnahmung des Computers ist allerdings schon nicht unproblematisch, wenn einer solchen Subsumption ein Medienbegriff zugrunde liegt, der spezifischen Eigenschaften des Computers nicht gerecht wird. Erst ein für beide Bereiche tragfähiger Medienbegriff erlaubt auch die wechselseitige Bezugnahme, Anschlussfähigkeit und die sinnvolle interdisziplinäre wissenschaftliche Diskussion (zur Problematik der Unterschiedlichkeit von Medienbegriffen schon allein in deutschsprachigen Kompetenzmodellen vgl. z. B. Welling et al. 2013).

Aus pädagogischer Perspektive lässt sich der Medienbegriff über die (Erfahrungs-) Formen entwickeln, in denen wir mit unserer Umwelt in Kontakt treten. Unterscheiden lassen sich reale Begegnungen mit Sachverhalten oder Personen, modellhafte Formen (z. B. ein Modell einer technischen Anlage), abbildhafte Formen (z. B. Fotos, Filme oder animierte Darstellungen) und symbolische Formen (z. B. gesprochene Sprache oder schriftliche Texte). Da diese Erfahrungsformen in gewisser Weise einen vermittelnden Charakter haben, werden sie manchmal schon selbst als Medien bezeichnet. Für die Medienpädagogik erscheint es zweckmässiger, den Medienbegriff auf technisch vermittelte Erfahrungsformen einzugrenzen (vgl. Tulodziecki et al. 2010, 27 ff.). Dies eröffnet in besonderer Weise die Möglichkeit, die Merkmale technisch vermittelter Erfahrungen und Inhalte zu untersuchen und wissenschaftliche Aussagen dazu zu formulieren. Beispiele für Medien in diesem Verständnis sind Buch, Zeitung, Film und Fernsehen, Radio, Video sowie Computer und Internet. Die Erfahrungsformen sind damit auf bildhafte und symbolische Formen eingeschränkt und beruhen auf Zeichengebrauch. Allerdings ist beispielsweise ein Buch als blosser Gegenstand noch kein Medium, sondern wird erst durch die kommunikationsbezogene Absicht und Nutzung bzw. durch die kommunikativen Zusammenhänge, in denen es steht, zu einem Medium. Zugleich bedeutet dies, dass der Medienbegriff sowohl die technischen Geräte bzw. Einrichtungen zur Übertragung, Speicherung, Wiedergabe oder Verarbeitung von Zeichen als 
auch die dazugehörigen Materialien bzw. die Software sowie deren funktionales Zusammenwirken bei der Kommunikation umfasst (vgl. Tulodziecki/Herzig 2002, 64). Medien können somit als Mittler verstanden werden, durch die in kommunikativen Zusammenhängen (potenzielle) Zeichen mit technischer Unterstützung übertragen, gespeichert, wiedergegeben oder verarbeitet und in abbildhafter oder symbolischer Form präsentiert werden (vgl. zur Genese dieser Definition Herzig 2012).

Ein solcher Medienbegriff rekurriert also nicht nur auf das Artefakt als Gegenstand, auf die vermittelnde Funktion und die kommunikative Situation, sondern auch auf die Bedeutung der technischen Bedingtheit dessen, was sich uns als Medienangebot zeichenbasiert offeriert (oder wir selbst als solches gestalten). Auf die besonderen Eigenschaften computerbasierter Medien wird in der genannten Definition mit der Funktion der Verarbeitung von Zeichen hingewiesen (zur Präzisierung der Sprechweise der Zeichenverarbeitung vgl. Herzig 2012, 102 ff.). Digitale, d. h. computerbasierte Medien weisen das Alleinstellungsmerkmal auf, Zeichen prozessieren und verarbeiten zu können - sie beruhen auf Rechenmaschinen, die Gegenstand der Informatik sind. Dies müsste definitorisch keine Berücksichtigung finden, wenn die technische Basis Digitaler Medien für pädagogische Fragen nicht relevant wäre, d. h. wenn die (medien-)pädagogische Auseinandersetzung sich auf die wahrnehmbaren Oberflächenphänomene des Artefakts beschränken könnte. Gerade dies ist nicht der Fall. Umgekehrt müsste der Computer als Medium keine Berücksichtigung finden, wenn es nur um die zeichenverarbeitende Funktion ginge und der Computer in der Interaktion und Kommunikation des Menschen mit der (Um-)Welt keine Rolle spielte. Auch dies ist gerade nicht der Fall.

Die hier vorgeschlagene Definition beinhaltet Setzungen, die grundsätzlich auch anders erfolgen könnten, aber als solche nicht vermeidbar sind. Auf die Unmöglichkeit, eine «bündige Antworts zu finden und auf die mit einer Definition verbundenen vielfältigen, inneren Spannungen, denen insbesondere vielschichtige Mediendefinitionen aufgrund unterschiedlicher Theoriebezüge unterliegen, weist auch Winkler hin (vgl. 2004, 9). In seinem eigenen - medienwissenschaftlich motivierten - weiten Definitionsvorschlag sieht er Medien als Maschinen gesellschaftlicher Vernetzung (Kommunikation), die zeichenbasiert symbolisches Probehandeln ermöglichen, dem Kommunizierten (Inhalt) eine Form auferlegen, auf Technik(en) und Praxen angewiesen sind, Raum und Zeit überwinden und aufgrund selbstverständlicher Nutzung tendenziell unsichtbar(er) werden (vgl. ebd., 10 ff.). Im Vergleich zur hier skizzierten Definition zeigen sich viele Anschlüsse, wenngleich unter den Medienbegriff von Winkler aufgrund eines Körpertechniken und Praxen einschliessenden Technikbegriffs auch körperliche Medien fallen. 


\section{Intradisziplinäre Sichtweisen}

In welcher Form Computer bzw. Informations- und Kommunikationstechnologien vor dem Hintergrund eines interdisziplinären Medienbegriffs - in der wissenschaftlichen Auseinandersetzung innerhalb der Medienpädagogik und der Informatik (Ebene der Objekttheorien) eine Rolle spielen, soll im Folgenden skizziert werden.

\section{Medienpädagogische (Selbst-)Verständnisse}

Betrachtet man die Entwicklung von medienpädagogischen Konzepten, so ist in Bezug auf die mediendidaktischen Entwicklungen festzustellen, dass jeweils «neue` Medien - z. B. Lehrmaschinen im programmierten Unterricht, das Schulfernsehen oder computerbasierte Medien - zwar verschiedene Einflüsse auf die didaktische Diskussion, d. h. die Vorstellungen zur Gestaltung von (unterrichtlichen) Lehr- und Lernprozessen, gehabt haben, allerdings nicht zu einer expliziten Auseinandersetzung mit technischen Grundlagen Anlass gaben (vgl. z. B. Hagemann 2001, 32 ff.). Allenfalls in der (eigenen) Gestaltung von Medienangeboten, z. B. unter Verwendung von Computerprogrammen, ist die funktionale Beherrschung von technischen Anwendungsgrundlagen erforderlich. Neben Gestaltungsaspekten richtet sich die pädagogische - und primär auch (lern-)psychologische - Diskussion vor allem auf die empirische Untersuchung von Effekten spezifischer Medienmerkmale auf das Lernen (vgl. Tulodziecki/Herzig 2004, 76 f.; Herzig 2014).

In Bezug auf medienerzieherische Ansätze lässt sich - u.a. als Reaktion auf die Entwicklung technischer Artefakte von der Massenpresse über die Filmtechnik und den Rundfunk als Radio und Fernsehen - eine Entwicklung von bewahrpädagogischen bis handlungsorientierten Ansätzen nachzeichnen (vgl. Tulodziecki et al. 2010, 157 ff.). Die technischen Grundlagen der Medien spielten dabei allerdings nur eine funktionale Rolle. So ist beispielsweise die Technik des Fernsehgerätes für die Fernseherziehung, die sich im Wesentlichen auf den Umgang mit Inhalten bezieht, nicht relevant, bestenfalls für die pädagogische Begründung der vermeintlichen Schädlichkeit z. B. des schnellen Zeilenwechsels von Kathodenstrahlröhren und der damit verbundenen Sakkadenbewegungen der Augen (vgl. z. B. Patzlaff 2001). Auch in Bezug auf andere Artefakte sind technische Aspekte ebenfalls eher funktional bedeutsam, z. B. in der aktiven Medienarbeit, wenn es in der Fotografie um das Verständnis von Blende oder Belichtungszeiten geht und dazu die Auseinandersetzung mit physikalisch-optischen Grundlagen erforderlich ist. Auch die Entwicklung des Computers bzw. seine Verbreitung in vielen gesellschaftlichen Bereichen wurde in der medienpädagogischen Diskussion ausführlich und kontinuierlich reflektiert. Analysiert man entsprechende Konzepte, so findet sich durchgängig eine Aufnahme bzw. «Integration`von informations- und kommunikationstechnologischen Aspekten (zur Übersicht vgl. Herzig 2012, 43 ff.; Zorn 
2011, 188). Die theoretischen Bezugsrahmen in medienpädagogischen Ansätzen sind unterschiedlich und reichen - ohne Anspruch auf Vollständigkeit - von Veränderungen kommunikativer Handlungen durch Technik (vgl. z. B. Baacke 1997; 1999) über die Erweiterung von Kulturtechniken (vgl. z. B. Doelker 1992) und semiologische Konzepte der Cultural Studies (vgl. z. B. Moser 2010) bis hin zu sozio- und kulturtechnischen (vgl. Wagner 2013), systemtheoretischen (vgl. Spanhel 2006) und bildungstheoretischen Konzepten (vgl. z. B. Jörissen/Marotzki 2009; Sesink 2013). Diese teilweise transdisziplinären Ansätze beziehen sich aber in der Regel nicht auf eine explizite Auseinandersetzung mit informatischen Grundlagen, sondern fokussieren auf die genannten Schwerpunkte.

\section{Informatische (Selbst-)Verständnisse}

Eine erste Bezugnahme der Informatik (genauer: eines Interessenverbandes) auf die Medienerziehung erfolgte Ende der 1980er Jahre, als sich parallel zu medienpädagogischen Konzepten Ansätze einer Informationstechnischen Grundbildung (ITG) entwickelten, die von der Bund-Länderkommission für Bildungsplanung und Forschungsförderung in einem Gesamtkonzept für die informationstechnische Bildung formuliert wurde und sich auf anwendungsbezogene, technische, algorithmische und gesellschaftliche Aspekte bezieht (BLK 1987). Die Beweggründe für eine solche Grundbildung sind insbesondere in Entwicklungen im Bereich der Mikroelektronik zu sehen, weil diese als entscheidende Schlüsseltechnologie für die Zukunft galt. Berührungspunkte zwischen der Medienerziehung und der Informationstechnischen Grundbildung entstanden vor allem durch folgende Entwicklungen: erstens die Tatsache, dass die Computertechnologie auch für die herkömmlichen Medien zunehmend wichtiger wurde, zweitens die Übernahme medialer Funktionen durch den Computer und drittens die Forderung, die Auseinandersetzung mit der Computertechnologie im Rahmen von Erziehungs- und Bildungsaufgaben zu diskutieren. Demgemäss wurde auch in bildungspolitischen Verlautbarungen der 1990er Jahre eine Verbindung von Informationstechnischer Grundbildung und Medienerziehung nahe gelegt (vgl. z. B. KMK 1997). Eine erste explizite Verbindung der beiden Bereiche findet sich in der Empfehlung der Gesellschaft für Informatik (GI) von 1999, in der es heisst:

Der spezifische Beitrag der informatischen Bildung zur Medienerziehung liegt in der Bereitstellung grundlegender informatischer Methoden und Sichtweisen, die ein Verständnis des Mediums Computer bzw. computerbasierter Medien ermöglichen. Dieser Beitrag kann von keinem anderen Bildungsangebot geleistet werden. (1999, VI)

Gleichzeitig wird auf die Notwendigkeit eines festen Platzes der Informatik in den Unterrichtsfächern hingewiesen. In der Umsetzung hat sich das integrative 
Konzept letztlich nicht bewährt. Insbesondere die über Anwendungsfertigkeiten hinausgehenden Zielvorstellungen konnten nicht in angemessener Form erreicht werden (vgl. Wilkens 2000, 28), zudem zeigten sich deutliche Schwierigkeiten in der Konkretisierung und Abgrenzung eines eigenständigen Inhalts- und Gegenstandsbereiches und die Verortung innerhalb der Fächer, die einen Beitrag zu den informationstechnischen Bildungszielen leisten sollen (vgl. Herzig 2012, 38 f.). Aber auch eine konsequente Einbettung informationstechnischer Bildung in Medienbildungskonzepte ist unterblieben - sowohl konzeptionell als auch auf praktischer Ebene. Zwar haben einzelne Bundesländer Ansätze entwickelt, beide Bereiche umzusetzen, aber auch diese Versuche verbleiben eher auf einer parallelen denn integrativen Ebene. Letztlich ist dies auch Ausdruck der fehlenden Bestimmung dessen, was integrativ oder interdisziplinär ist (vgl. ebd., 71).

Die Diskussion zwischen Medienerziehung und ITG war von Aussen angestossen und nicht Ausdruck einer intradisziplinären Klärung des Selbstverständnisses der Informatik - die bis heute nicht wirklich abgeschlossen scheint. Klärungen des Wesens der Informatik erfolgen zum einen über Hinweise auf die automatisierte Informationsverarbeitung oder über die Teildisziplinen, z. B. die angewandte, theoretische, technische oder praktische Informatik (vgl. z. B. Gumm/Sommer 2009). Allerdings wird an vielen Stellen auch auf die Querschnittsfunktionen und die Reichweite der Informatik in unterschiedliche wissenschaftliche und gesellschaftliche Bereiche verwiesen. Explizite Verbindungen zum Computer als Medium finden sich heute am ehesten in der Medieninformatik, die sich als interdisziplinär versteht und mit der Analyse und Konstruktion multimedialer Technologien und Anwendungen auf Grundlage informatischer Systeme beschäftigt (vgl. Herczeg 2007, 9). Pädagogische Anschlüsse kommen hier insbesondere in Bezug auf die Gestaltung von (interaktiven) Lernmedien zum Tragen, darüber hinaus finden sich auch explizite Erörterungen der moralischen oder ethischen Implikationen von Digitalen Medien, wenn allerdings auch nicht in einem medienpädagogischen Zusammenhang (vgl. ebd., 205 ff.).

\section{Interdisziplinäre Sichtweisen}

Die Beschäftigung von Informatik und Pädagogik mit Computern resp. Medien zeigt - vor dem Hintergrund der bisherigen Überlegungen - auf der Ebene der entsprechenden disziplinären Ansätze Bezugspunkte und Schnittstellen. Darüber hinausgehende interdisziplinäre Aspekte finden sich - ebenfalls ohne Anspruch auf Vollständigkeit - beispielsweise in den Arbeiten von Schelhowe, Zorn oder Herzig. Die explizite Bezugnahme auf informatische Aspekte ist dabei durch die (Medien-)Bildungsperspektive motiviert. 
Schelhowe (2006) argumentiert, dass die Computeranwendungen die Nutzer immer weniger dazu zwingen, sich auf die Prozesse der Maschine zu konzentrieren. Der Computer als zeichenverarbeitende Maschine ist an der Produktion und Veränderung medialer Inhalte beteiligt. Er ist kein 'geräuscharmes〉 Medium, das das, worauf es angewendet wird, so wenig wie möglich verändert (vgl. Esposito 1993, 339). Dies bedeutet, dass das Verständnis dessen, was technisch im Computer passiert, für die Auseinandersetzung mit computervermittelten medialen Inhalten bedeutsam ist: «Programme, die der Welt des Semiotischen, der Zeichen angehören, wirken direkt und ohne menschliche Vermittlung auf die physikalische Realität - und das wird für die Anwendungen der Zukunft immer mehr der Fall sein» (ebd., 7 f.). Wesentliche, d. h. im Wesen des Artefakts angelegte, Merkmale des Computers, die in Bildungsprozessen offengelegt werden können, sind «die der zunehmenden Semiotisierung aller unserer Arbeits- und Lebensprozesse und der Kondensierung und Reduzierung von Zeichenprozessen in Modellen, Formalisierungen und schließlich in Implementierungen in Automaten» (Schelhowe 2007, 175).

In ähnlicher Weise kommt auch Zorn zu der Einschätzung, dass eine Auseinandersetzung mit der ästhetischen und phänomenologischen Seite der Digitalen Medien nicht ausreiche, sondern dass es zum Verständnis der Logik und der Wirkungsweisen auch «der Perspektiven und Erkenntnisse der Informatik und Softwaretheorie» bedarf (2011, 182). Dazu zählen Prozesse der Semiotisierung, der Algorithmisierung und der Berechenbarkeit, die weitreichende kulturelle Auswirkungen nach sich ziehen. Vor diesem Hintergrund plädiert Zorn für die Herausbildung einer spezifischen Perspektive auf Digitale Medien, «in der sowohl für Konzepte der Medienkompetenz als auch solche von Medienbildung die technischen Grundlagen berücksichtigt werden» (ebd., 199).

Herzig zeichnet mit Hilfe semiotischer Überlegungen die Entwicklung der Medien als technische Artefakte zur Registration, Produktion, Transmission und Berechnung von zeichenfähigen Mustern nach und arbeitet auf dieser Basis die Besonderheiten Digitaler Medien heraus (vgl. 2012, 139 ff.). Dabei werden auch informatische Prozesse, z. B. die Softwareentwicklung oder die Mensch-Maschine-Interaktion, als Zeichenprozesse rekonstruiert und damit als anschlussfähig an die medienpädagogische Diskussion im Rahmen kommunikations- und interaktionstheoretischer Überlegungen charakterisiert (vgl. ebd., 118 ff.; 177 ff.).

Diese Arbeiten sind hier zunächst nicht systematisch, sondern heuristisch im Sinne einer Spurensuche ausgewählt. Sie sollen Perspektiven einer interdisziplinären Sicht eröffnen, in der eine integrative Form von Medienbildung präferiert wird, ohne dabei die Eigenständigkeit der Informatik und der Medienpädagogik als Disziplinen in Frage zu stellen. Gleichzeitig erfolgt eine explizite Auseinandersetzung mit technischen Grundlagen der Informatik, die Anschlüsse an die medienpädagogische Diskussion erlauben. 


\section{Ideen und Prinzipien im interdisziplinären Raum}

Ausserhalb der medienpädagogischen Diskussion finden sich auch in den Medienwissenschaften und der Informatik selbst Überlegungen zu grundlegenden Ideen und Prinzipien, die den interdisziplinären Raum ‘füllen` können. Im Folgenden sollen - wiederum in heuristischer Weise - einzelne Arbeiten angesprochen werden, um - ohne Anspruch auf Vollständigkeit und Systematik - Hinweise auf Ideen und Prinzipien offen zu legen.

Aus einer medien- und kulturwissenschaftlichen Analyse heraus unterscheidet Manovich fünf Prinzipien neuer Medien. Mit der numerischen Repräsentation bezeichnet er die Formalisierbarkeit und damit auch die Programmierbarkeit (vgl. 2001, 27 ff.) der Medien, d. h. ihre algorithmische Manipulation. Die Modularität als Prinzip hebt darauf ab, dass Medienobjekte stets als diskrete Muster bzw. als Kollektionen von diesen vorliegen (vgl. auch den Musterbegriff bei Herzig 2012, 139 ff.). Ohne ihre eigenständige Struktur zu verlieren, können Sie - fraktalähnlich - in grössere Objekte integriert werden. Als Folge dieser beiden Grundprinzipien ergeben sich die automatische Verarbeitung von Medienobjekten, die Variabilität und die Transkodierbarkeit. Mit Variabilität ist die Erstellung von verschiedenen Versionen ein und desselben Medienobjekts gemeint, während sich «transcodings auf die Umwandlung von Medienobjekten in Datenstrukturen bezieht. Später fokussiert Manovich diese Überlegungen noch einmal und hebt die Bedeutung von Software zum Verständnis von Medien hervor. Die besondere Eigenschaft des Computers als «Metamedium» sei die Simulation verschiedener Medien in bzw. mithilfe von Software. Dabei geht er von folgender Grundidee aus: Medienobjekte werden durch Computer in Datenstrukturen überführt bzw. in solchen codiert, z. B. eine Buchseite als Textfile (wobei der Begriff der Datenstrukturen hier eine höhere Aggregationsebene als in der Informatik meint; vgl. 2013, 201). Die Software simuliert die physikalischen, mechanischen oder elektronischen Techniken der $\mathrm{Na}$ vigation, Erstellung, Bearbeitung oder Interaktion mit den Daten (media data). Für das Beispiel der Buchseite werden also Techniken des Schreibens und Editierens oder des Formatierens und der Präsentation simuliert. Eine solche «softwarization) des Mediums führt zu der Frage, was das Medium dann eigentlich sei. Manovich beantwortet dies mit der Formel «medium = algorithms + a data structure» (ebd., 207). Der Computer als Hybridmedium lasse auch die Unterscheidung von alten und neuen Medien durch das Digitale nicht mehr sinnvoll erscheinen, weil die spezifischen Eigenschaften und Qualitäten neuer Medien keine den Medien inhärente Eigenschaften seien, sondern weil die digitale Repräsentation dazu führe, dass die Daten durch Computer bearbeitbar werden, aber «it is the software that determines what we can do with them» (ebd., 149), d. h. «there is no such thing as «digital media». There is only software - as applied to media (or 〈content))» (ebd., 152). 
Aus der Perspektive von «Informatik und Gesellschaft» beschreibt Keil Software als den eigentlichen Betrachtungsgegenstand der Informatik und charakterisiert sie als «typografische Steuerungen, d. h. Signale und Signalwege werden als Text beschrieben, der von einem Prozessor ausgeführt wird» $(2011,166)$. Die Eigenschaft von Software in ihrer Dualität als einerseits ausführbare Maschinensteuerung und andererseits flexibles Modellierungsinstrument «hat zur Folge, dass in Software menschliches Verhalten und Verhältnisse in einem Maße widergespiegelt werden wie in keinem anderen technischen Artefakt» (ebd.). Software lässt sich demnach auf der Basis zeichentheoretischer Grundlagen in Verbindung mit den Prinzipien der Formalisierbarkeit und Berechenbarkeit beschreiben.

Mit dem Ansatz des computational thinking bezeichnet Wing (2006; 2010) eine universell anwendbare analytische Fähigkeit neben den Kulturtechniken des Lesens, Schreibens und Rechnens: «[...] computational thinking describes the mental activity in formulating a problem to admit a computational solution. The solution can be carried out by a human or machine, or more generally, by combinations of humans and machines» $(2010,1)$. Diese Denkweisen sind also nicht auf die Informatik beschränkt, sondern spielen sowohl im Alltag als auch in allen Wissenschaften eine Rolle - etwa als Sortieralgorithmen oder divide-and-conquer-Strategien. Dementsprechend stehen auch nicht die Artefakte im Vordergrund, sondern die Konzepte, zu denen neben Algorithmen auch Datenstrukturen, Zustandsmaschinen, Sprachen, Logik und Semantik usw. zählen. Explizit nicht zu den grundlegenden allgemeinbildenden Ideen und Denkweisen gehören nach Wing Anwendungskenntnisse (computer literacy) und Programmierfähigkeiten.

Fundamentale Ideen finden sich auch bei Denning, der als Informatiker in seinem Plädoyer für die Betrachtung der Informatik als Naturwissenschaft Kategorien von "great principles of computing" unterscheidet, die die wesentlichen Funktionen von Computersystemen erfassen sollen: Berechnung (Bedeutung und Grenzen von Berechnungen), Kommunikation (zuverlässige Übertragung von Daten), Koordination (Kooperation zwischen vernetzten Entitäten), Erinnerung (Speicherung und Auffinden von Informationen), Automatisierung (Bedeutung und Begrenzung von Automatisierung), Evaluation (Leistungsprognose und Kapazitätsplanung) und Design (Entwicklung zuverlässiger Softwaresysteme) $(2007,15)$. Diese fundamentalen Prinzipien sind - wie auch das computational thinking - nicht auf die Informatik beschränkt, sondern für viele andere Bereiche konstitutiv und sollen eine gemeinsame Sprache begründen, um ‘computing) in anderen Disziplinen zu diskutieren. Gleichzeitig sieht Denning sie als inspirierend für Lehren und Lernen an, «where it is producing innovative ways to teaching computing and is inspiring young people to consider computing majors» (ebd., 17).

Mit dem Ziel, unabhängig von aktuellen Strömungen den Kern der Informatik zu fassen und für die Schule aufzubereiten, hat Schwill aus der Perspektive der 
Didaktik der Informatik nach fundamentalen Ideen gefragt. Sie entsprechen Denk, Handlungs-, Beschreibungs- oder Erklärungsschemata und müssen verschiedenen Kriterien genügen - der Breite der Anwendbarkeit, Bezügen zur Alltags- und Lebenswelt oder der Vermittelbarkeit auf unterschiedlichen kognitiven Anforderungsniveaus (vgl. Schubert/Schwill 2011, 64 f.). Als Masterideen nennt Schwill die strukturierte Zerlegung, die Sprache (bzw. Formalisierung) und die Algorithmisierung (vgl. ebd., 68 ff.). Auf notwendige Ergänzungen der Digitalisierung und der Interaktivität weist Engbring (2012) hin.

Die Beispiele zeigen, dass in den interdisziplinären Diskussionen auf zentrale Begriffe, Prinzipien oder Paradigmen - wie z. B. Zeichen, Semiotisierung, Muster, Berechenbarkeit, Formalisierung, Software, Algorithmus und Interaktion - rekurriert wird, die sowohl im medienpädagogischen als auch im medienwissenschaftlichen und informatischen Diskurs anschlussfähig sind. Unabhängig davon, ob es sich hier schon um eine vollständige oder trennscharfe Liste von Prinzipien handelt, liegt der Wert solcher Konzepte in ihrem Beitrag zur theoretischen Fundierung eines interdisziplinären Bereiches. In Bezug auf Digitale Medien können über diese fundamentalen Konzepte die Prozesse der Entwicklung von Software, die technisch-physikalischen Grundlagen der maschinellen Repräsentation von Software, Prozesse der maschinellen Verarbeitung und die Interaktion mit Software über Medien als Artefakte beschrieben und erklärt werden. Aus (medien-)pädagogischer Perspektive ist dabei insbesondere bedeutsam, dass die durch Software gesteuerten Prozesse Inhalte verändern bzw. an der Erzeugung von (neuen) Inhalten beteiligt sind (vgl. Abb. 1). Auf diesen Sachverhalt haben z. B. Schelhowe (2007) und Sesink (2009) hingewiesen. Sesink argumentiert, dass die Lernobjekte eine spezifische Transformation erfahren haben, über die Lernende angeregt werden müssen nachzudenken (Medialitätsbewusstsein). Dem Lernenden treten nicht die Dinge, sondern die Theorie dieser Dinge in einer durch die Medientechnik bestimmten Erscheinungsform entgegen (vgl. ebd., 215; zum Medialitätsbewusstsein vgl. auch Wagner 2013).

Auf der Basis der genannten grundlegenden informatischen Prinzipien lassen sich die Semiotisierung von Welt bzw. auf sie bezogene Gegenstandsbereiche und Objekte als Voraussetzung für ihre Formalisierbarkeit als zentrale Grundideen computerbasierter Medien beschreiben. Gleichzeitig wird der Wechsel von der symbolischen auf die maschinelle Ebene durch semiotische und technische Kategorien geschlossen und als maschinisierte Durchführung von Berechnungen auf der Basis von in Software implementierten algorithmischen Schritten verstehbar. Die Tatsache, dass maschinelle Prozesse letztlich sinnentleerte Prozesse sind, macht ausserdem deutlich, dass im Laufe der Entwicklung von Informatiksystemen (d. h. im Wesentlichen der Entwicklung von Software) Kontextwissen erst dekontextualisiert und ausgelagert und im Interaktionsprozess wieder rekontextualisiert werden 
muss (vgl. Herzig 2012, 129 f.). Dies gilt auch für die durch Software verarbeiteten Daten bzw. genauer als Daten bezeichneten Zeichenaspekte.

Zur Fundierung eines Brückenschlags zwischen Medienbildung und Informatischer Bildung oder Medienpädagogik und Informatik besteht also - wie angedeutet bereits eine Reihe von zielführenden Vorschlägen. Sie reichen von der technischen Ebene (Maschine) über die symbolische Ebene (Software) bis hin zur kognitiven Ebene ((Denk-)Strukturen) und deren Wechselwirkungen. Was noch weitgehend aussteht, ist die konsequente Auswertung und ggf. Zusammenführung dieser Vorschläge hinsichtlich ihres Beitrags oder ihrer Notwendigkeit zur Realisierung einer aktiven und aufgeklärten gesellschaftlichen Partizipation und Mitgestaltung sowie der Ermöglichung persönlicher Entwicklung.

\section{Interdisziplinarität im Bildungsdiskurs}

Auf der Basis der vorgenannten grundlegenden Ideen und Prinzipien lässt sich die Entwicklung der Medien als technische Artefakte sowohl in analoger als auch in digitaler Form herleiten und nachzeichnen. Dabei geht es nicht nur um eine historische Rekonstruktion, sondern um den Aufweis von Entwicklungen, die grundlegende Veränderungen im Verhältnis des Menschen zu sich selbst, seiner dinglichen und sozialen Umwelt bewirken. Mit anderen Worten, das Verständnis dieser Prozesse hat eine aufklärende und bildende Wirkung. Im Verlauf der bisherigen Argumentation wurde zunächst auf die strukturelle und inhaltliche Konkretisierung interdisziplinärer Zugänge abgehoben. Daran schliesst sich die Frage an, wie interdisziplinäre Grundlagen in den Bildungsdiskurs, insbesondere in Bildungspläne oder Lehrpläne Eingang finden.

Diese Diskussion kann disziplinär sowohl mit Bezug auf die Informatik als auch die Medienbildung als Unterrichtsfach geführt werden. Für die Medienbildung ist diese Frage - mit Ausnahme einzelner Realisierungen als eigenständige Lernbereiche - zugunsten einer Querschnittsaufgabe entschieden (vgl. KMK 2016). Bildungspolitisch finden sich auf allen Ebenen hinreichend Plädoyers, Empfehlungen, Forderungen etc. in Bezug auf die schulische Entwicklung von Medienkompetenz oder die Umsetzung von Medienbildung, allerdings wird bisher keine konsequente, d. h. verbindliche Umsetzung sichergestellt. Medienbildung ist weitestgehend und im besten Fall immer noch eine Option oder ein Angebot. Für die Informatik lässt sich auf einen langen Diskurs über den allgemeinbildenden Wert des Faches verweisen, insbesondere die Etablierung eines obligatorischen Schulfaches (vgl. z. B. Steffen 2003; Humbert 2011; GI 2015; Knobelsdorf et al. 2015). Aber auch hier ist bislang kein verpflichtendes Fach in allen Bundesländern eingeführt (s. u.).

Da es in diesem Beitrag um Interdisziplinäres geht, wäre ein neuer Querschnittsbereich plausibel, der weder Informatische Bildung noch Medienbildung heisst. Da 
alle Wortschöpfungen für einen solchen Bereich auch wiederum mit Beschränkungen, möglichen Missverständnissen und ggf. Schieflagen verbunden wären, wird im Folgenden - im Bewusstsein der Problematik - von Medienbildung gesprochen. In Bezug auf die Umsetzung einer solchen - interdisziplinären - Medienbildung erscheint die Einbettung in die Diskussion um Kompetenzen und Bildungsstandards geboten, da so wichtige Anschlussstellen an die schulpädagogische Diskussion und den kompetenzorientierten Unterricht hergestellt werden können.

Dazu bedarf es zweier Schritte: Zunächst muss ein übergreifendes Kompetenzmodell für den Querschnittsbereich Medienbildung entwickelt werden, dann ist die Frage zu klären, welche Fächer die Entwicklung der entsprechenden Kompetenzen sicherstellen können oder sollen.

Unter dem Begriff der Medienkompetenz wird eine inzwischen lang andauernde Debatte über notwendige oder wünschenswerte Fähigkeiten, Fertigkeiten und Einstellungen von Kindern und Jugendlichen geführt. Die Vorstellungen reichen dabei von relativ grundlegenden Zielkatalogen bis hin zu ausdifferenzierten Kompetenzmodellen, in denen Dimensionen, Bereiche und Niveaustufen unterschieden werden. In exemplarischer Weise sollen hier drei Modelle im Kontext des Zusammenhangs von Medienbildung und Informatischer Bildung angesprochen werden.

- Ein handlungs-, entwicklungs- und bildungstheoretisch begründetes Modell haben Tulodziecki, Herzig und Grafe vorgelegt, das neben zwei Grundformen bzw. Handlungszusammenhängen im Medienbereich drei Inhaltsbereiche unterscheidet und daraus fünf Kompetenzbereiche ableitet (vgl. 2010, 180 ff.): Auswählen und Nutzen von medialen Angeboten, Gestalten und Verbreiten eigener medialer Beiträge, Verstehen und Bewerten von Mediengestaltungen, Erkennen und Aufarbeiten von Medieneinflüssen, Durchschauen und Beurteilen von Bedingungen der Medienproduktion und Medienverbreitung. Die einzelnen Bereiche sind weiter ausdifferenziert, z. B. geht es im letztgenannten Bereich um technische, ökonomische, rechtliche, personale und institutionelle sowie um politische und gesellschaftliche Bedingungen. Innerhalb der Teilbereiche sind dann auf verschiedenen Niveaustufen Kompetenzerwartungen als Standards formuliert. Dass ein solches Modell auf der Ebene von Dimensionen und Bereichen nicht explizit informatische Kompetenzen nennt, liegt zum einen in der Perspektive eines - alle Medien - umfassenden Modells begründet, zum anderen in der Granularität der Bereiche. Nimmt man beispielsweise das Phänomen der Personalisierung von Ergebnissen in Suchmaschinen, so lässt sich dies unter ökonomischen und technischen Bedingungen thematisieren. Technisch bedeutet dies zu fragen, welche informatischen Aspekte ein Verständnis und eine Einschätzung (auch im Sinne der persönlichen Handlungsregulation) der zugrunde liegenden Phänomene ermöglichen (s. u.) oder anders 
ausgedrückt, welche Kompetenzen im Hinblick auf den interdisziplinären Bereich in das Modell aufgenommen werden sollten.

- Auf die zur persönlichen Entwicklung und Orientierung sowie zur beruflichen Entfaltung notwendigen Kompetenzen in einer digital geprägten Kultur heben die Empfehlungen einer BMBF-Expertenkommission ab, die explizit Perspektiven der Informatik und der Medienbildung integriert (vgl. BMBF 2010). Hier werden die Themen- bzw. Aufgabenfelder Information und Wissen, Kommunikation und Kooperation, Identitätssuche und Orientierung sowie Digitale Wirklichkeiten und produktives Handeln unterschieden (vgl. ebd., 8 ff.). Die bei der Formulierung von Teilkompetenzen zugrundeliegenden Handlungsdimensionen beziehen sich auf die Nutzung, kritische Beurteilung, Gestaltung, Interaktion und Partizipation mit Medien sowie auf die Nutzung von Medien als Lernmittel. Insgesamt werden technische, ökonomische, politische, rechtliche, ethische, und ästhetische Aspekte einbezogen.

- In dem der internationalen Studie zu computer- und informationsbezogenen Kompetenzen von Schülerinnen und Schülern (ICILS) zugrunde liegenden Kompetenzmodell werden die Teilbereiche Informationen sammeln und organisieren sowie Informationen erzeugen und austauschen unterschieden. Die Bereiche ihrerseits werden noch einmal in Aspekte ausdifferenziert, z. B. Wissen über Computernutzung, Zugriff auf und Bewertung von Informationen, Verarbeitung und Organisation von Informationen (vgl. Senkbeil et al. 2014, 88 ff.).

Ein Vergleich dieser Modelle ist insofern aufschlussreich, als sie drei unterschiedliche Zugänge und Intentionen verfolgen. Während das - theoretisch-deduktiv begründete - medienpädagogische Modell sich auf alle Medien und umfassende Kompetenzanforderungen im Kontext spezifischer Bildungsziele richtet, fokussiert das zweite Modell auf Digitale Medien, wird allerdings in seiner Struktur- und Entwicklungslogik nicht weiter offen gelegt. Gleichwohl zeigt ein Vergleich der beiden Strukturen, dass die Modelle eine hohe Kompatibilität aufweisen, wenn sie auch inhaltlich anders gegliedert sind. Das ICILS-Modell steht stärker in der Tradition von literacy-Konzepten und rekurriert auf den Umgang mit Informationen und damit verbundene Anwendungsfähigkeiten. Es ist damit kein umfassendes Medienkompetenzmodell, ist aber einer empirischen Prüfung durch Umsetzung in einen entsprechenden Kompetenztest zugänglich gemacht.

Die Modelle stellen gleichzeitig Anforderungen an ein Medienkompetenzmodell dar, das sowohl medienpädagogische als auch informatische Aspekte integriert. Ein solches Modell sollte

- umfassend in Bezug auf Medien, theoretisch fundiert und begründet sowie in der Genese nachvollziehbar sein. Es sollte Angaben zu Dimensionen, Aspekten und niveaubezogenen Differenzierungen enthalten. 
- einen mittleren Abstraktionsgrad in der Beschreibung von Standards aufweisen, der einerseits eine Anwendung auf eine breite - grundsätzlich entwicklungsoffene - Palette von Medien zulässt, andererseits aber auch, ggf. durch weitere Konkretisierungen, einer empirischen Prüfung unterworfen werden kann.

- auf den zentralen Prinzipien, Strukturen oder Wissensbeständen aufbauen, die erforderlich sind, um die übergeordneten Bildungsaufgaben zu realisieren.

Die Entwicklung von Kompetenzmodellen ist auch in der medienpädagogischen Diskussion nicht ohne Kritik geblieben, insbesondere im Hinblick auf den Bildungsbegriff (vgl. z. B. Moser et al. 2011). An dieser Stelle sei nur darauf verwiesen, dass ein prozessbezogenes Bildungsverständnis einer Formulierung von Kompetenzerwartungen nicht widerspricht, im Gegenteil. Standards beschreiben Entwicklungspunkte im Sinne anzustrebender Ziele. Das Erreichen dieser Standards geschieht in Lern- und Bildungsprozessen, über die das reflexive Verhältnis des Menschen zur Welt ermöglicht wird. Diese Ermöglichung geschieht quasi kontinuierlich auf der Basis jeweils verfügbarer und genutzter Kompetenzen.

Wenn Medienbildung kein eigenes Fach darstellt, ist sie auf die Umsetzung innerhalb der etablierten Fächer angewiesen, ggf. unterstützt durch fachübergreifende Formen. Dies bedeutet, dass alle Fächer angehalten sind, über ihre Beiträge zur Umsetzung von Zielen der Medienbildung nachzudenken und diese Ergebnisse in die Ausgestaltung eines o.g. Orientierungsrahmens einfliessen zu lassen. Diese Diskussion ist nicht neu und in vielen (Kern-)Lehrplänen finden sich auch entsprechende Anforderungen. Im Sinne einer umfassenden Medienbildung gilt es, im Rahmen von Schulentwicklungsprozessen für Schülerinnen und Schüler kohärente und konsistente Lern- und Bildungsmöglichkeiten zu schaffen, die sich nicht in singulären Massnahmen erschöpfen. Dies bedeutet aber auch zweierlei Anforderungen an Lehrpersonen: Zum einen müssen sie über grundlegende medienpädagogische Kompetenz verfügen (vgl. Herzig et al. 2015), zum anderen müssen die in Bezug auf die Medienbildung notwendigen fachlichen Grundlagen vorhanden sein. Während die erste Anforderung in der Ausbildung von Lehrpersonen noch immer ein grundlegendes Desiderat markiert, kann die zweite in der Regel unterstellt werden. Wenn allerdings die informatischen Anteile fachfremd unterrichtet werden, ist die Kritik einer drohenden Trivialisierung oder Verwässerung der Informatik berechtigt. Mit anderen Worten: Die Umsetzung einer umfassenden Medienbildung ist auf eine explizite informatikdidaktische Expertise angewiesen. Vieles spricht dafür, diese aus einem allgemeinbildenden Fach Informatik zu gewinnen. 


\section{Fazit}

Für einen interdisziplinären Bereich der Medienbildung lassen sich hinreichend «Spuren` in der Medienpädagogik und in der Informatik - ergänzend auch in der Medienwissenschaft - finden.

Die digital geprägte Welt stellt sich Schülerinnen und Schülern in Alltag und Freizeit zunächst nicht in originär digitalen, sondern in medialen Erscheinungsformen dar. Dies bedeutet, dass sie mit unterschiedlichen Medienangeboten interagieren - und zwar auf einer Ebene, auf der das Digitale im Wesentlichen unsichtbar bleibt. Aus Gründen der Benutzerfreundlichkeit von (Digitalen) Medien(-angeboten) ist dies auch überaus wünschenswert, dass die technische Seite des Mediums nicht in Erscheinung tritt (und entsprechend keine weitgehenden technischen oder auch informatischen Kompetenzen erforderlich sind, um Digitale Medien nutzen zu können). Allerdings ist es aus dem Blickwinkel von Medienbildung aber auch erforderlich, das Digitale - im Sinne zugrunde liegender informatischer Grundkonzepte oder Grundideen - wieder sicht- und erfahrbar und damit verstehbar zu machen. Zentrale Prinzipien und Konstrukte einer solchen interdisziplinären auf Mediatisierung und Digitalisierung rekurrierenden - Medienbildung sind z. B. Zeichen, Semiotisierung, Muster, Berechenbarkeit, Formalisierung, De- und Rekonstruktion, Software, Algorithmus und Interaktivität. Das digitale Medium bzw. die damit verbundenen Medienangebote werden über das Interface wahrnehmbar, nutzbar und gestaltbar und erlauben über Vernetzung auch die Kommunikation und Kooperation mit anderen (als grundlegende Interaktionsfelder). Dafür sind Kompetenzen in der Handhabung der entsprechenden Angebote erforderlich (als elementare Handhabungs- und Nutzungsformen), die aber allein für eine auf Selbstbestimmung, Partizipation und soziale Verantwortung zielende Bildung nicht hinreichend sind. Neben einer - für Information und Lernen, Problembearbeitung und Erkenntnisgewinn sowie Unterhaltung und Spiel (als grundlegende Handlungsfelder) - sachgerechten und zielführenden Nutzung und Handhabung ist einerseits ein grundlegendes Verständnis von Software und damit verbundener Prozesse der Formalisierung, Algorithmisierung, Berechenbarkeit und maschinellen Verarbeitung und andererseits ein kritisches Urteilsvermögen in Bezug auf die Einflüsse und Auswirkungen der Erzeugung, Analyse und Verarbeitung von Daten erforderlich (vgl. Abb. 1; vgl. dazu auch das «Dagstuhl-Dreieck», GI 2016). 


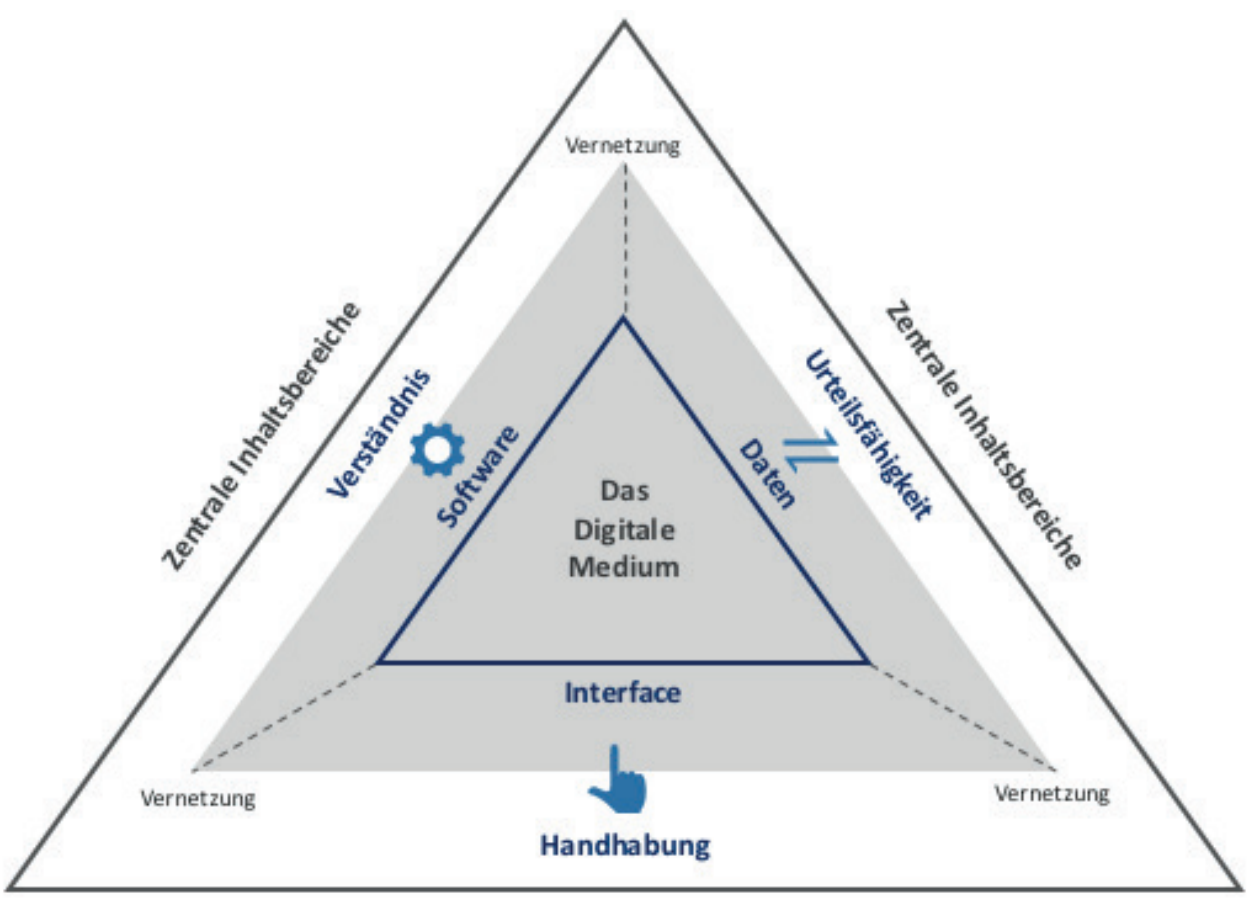

Handlungs- und Interaktionsfelder

Abb. 1.: Medienbildung - informatische und pädagogische Perspektiven.

Für die Gestaltung eines interdisziplinären Bereiches Medienbildung ist die Ausarbeitung der genannten Prinzipien in Verbindung mit einem entsprechend begründeten Kompetenzmodell erforderlich. Ein solches Modell sollte die Dimensionen der elementaren Nutzungs- und Handhabungsformen sowie der Handlungs- und Interaktionsfelder umfassen und auf Kenntnisse, Verstehen, Anwendungs- und Urteilsfähigkeit in zentralen Inhaltsbereichen zielen:

- Vielfalt, Struktur und Zugänglichkeit von Medienangeboten und Informatiksystemen,

- Möglichkeiten der Gestaltung von Medienangeboten und Interaktionsformen,

- Prozesse der (maschinellen) Erzeugung, Verarbeitung, Verbreitung und Auswertung von Zeichen bzw. Daten,

- Einflüsse von Medienangeboten und Informatiksystemen auf Realitätsvorstellungen, Emotionen und Verhalten sowie auf gesellschaftliche Prozesse,

- technische, rechtliche, ökonomische, personelle und institutionalisierte Bedingungen der Produktion und Verbreitung von Medienangeboten und Informatiksystemen.

Eine - vollständige oder teilweise - fächerintegrative Umsetzung ist auch auf Beiträge eines Unterrichtsfaches Informatik angewiesen. Dies bedeutet, dass es als allgemeinbildendes Fach in den Fächerkanon verpflichtend Einzug halten muss, 
wo dies noch nicht geschehen ist. Konsequenterweise muss dazu allerdings auch die Differenz zwischen einem informatischen Anteil zur Medienbildung und dem Unterrichtsfach Informatik herausgearbeitet werden.

\section{Literatur}

Baacke, Dieter. 1997. Medienpädagogik. Tübingen: Niemeyer.

Baacke, Dieter. 1999. «Medienkompetenz als zentrales Operationsfeld von Projekten». In Handbuch Medien: Medienkompetenz, Modelle und Projekte, hrsg. v. Dieter Baacke, Susanne Kornblum, Jürgen Lauffer, Lothar Mikos und Günther A. Thiele, 31-35. Bonn: Bundeszentrale für Politische Bildung.

BLK - Bund-Länder-Kommission für Bildungsplanung und Forschungsförderung. 1987. Gesamtkonzept für die informationstechnische Bildung (16). Bonn: Sekretariat der BLK.

BMBF - Bundesministerium für Bildung und Forschung, Hrsg. 2010. «Kompetenzen in einer digital geprägten Kultur. Medienbildung für die Persönlichkeitsentwicklung, für die gesellschaftliche Teilhabe und für die Entwicklung von Ausbildungs- und Erwerbsfähigkeit». http://www.dlr.de/pt/Portaldata/45/Resources/a_dokumente/bildungsforschung/ Medienbildung_Broschuere_2010.pdf.

Denning, Peter J. 2007. "Computing is a Natural Science». Communications of the ACM 50(7): 13-18.

Döbeli Honegger, Beat. 2013a. «Informatik ist mehr als Informatik! - Oder: Warum sich die Informatik mit dem Leitmedienwechsel befassen muss». In Informatik erweitert Horizonte, 15. GI-Fachtagung Informatik und Schule, hrsg. v. Norbert Breier, Peer Stechert und Thomas Willke, Bonn Gesellschaft für Informatik e.V., 11-20. Bonn: Köllen.

Döbeli Honegger, Beat. 2013b. «Informatik, ICT und Medienbildung». In informatik@gymnasium. Ein Entwurf für die Schweiz, hrsg. v. J. Kohlas, J. Schmid und C. A. Zehnder, 159-191. Zürich: Verlag Neue Zürcher Zeitung.

Doelker, Christian. 1992. «Medienpädagogik in der Sekundarstufe - der integrative Ansatz». In Medienpädagogisches Handeln in der Schule, hrsg. v. Wolfgang Schill, Gerhard Tulodziecki und Wolf-Rüdiger Wagner, 107-131. Opladen: Leske + Budrich.

Engbring, Dieter. 2012. «Zentrale Ideen der Informatik». In Ideen und Modelle, hrsg. v. M. Thomas und M. Weigend. 9-18. Norderstedt. Books on Demand GmbH.

Esposito, Elena. 1993. «Der Computer als Medium und Maschine». Zeitschrift für Soziologie (4): 338-354.

GI-Gesellschaft für Informatik. 1999. "Gl-Empfehlungen zur Informatischen Bildung und Medienerziehung». LOG IN 19(6): Beilage.

GI-Gesellschaft für Informatik. 2015. 3. Dagstuhl-Erklärung zur Informatik in der Schule. https://www.gi.de/fileadmin/redaktion/Download/GI-Dagstuhl-Erklaerung2015.pdf.

GI-Gesellschaft für Informatik. 2016. Dagstuhl-Erklärung: Bildung in der digital vernetzten Welt. https://www.gi.de/fileadmin/redaktion/Themen/dagstuhl-erklaerung-bildung-inder-digitalen-welt-2016.pdf

Gumm, Heinz-Peter, und Manfred Sommer. 2009. Einführung in die Informatik. München: Oldenbourg.

Hagemann, Wilhelm. 2001. «Von den Lehrmitteln zu den Neuen Medien». In Medien machen Schule, hrsg. v. Bardo Herzig, 19-55. Bad Heilbrunn: Klinkhardt.

Herczeg, Michael. 2007. Einführung in die Medieninformatik. München: Oldenbourg. 
Herzig, Bardo, Alexander Martin, Niclas Schaper, und Daniel Ossenschmidt. 2015. «Modellierung und Messung medienpädagogischer Kompetenz - Grundlagen und erste Ergebnisse». In Kompetenzerwerb an Hochschulen: Modellierung und Messung. Zur Professionalisierung angehender Lehrerinnen und Lehrer sowie frühpädagogischer Fachkräfte, hrsg. v. Barbara Koch-Priewe, Anne Köker, Jürgen Seifried und Evelin Wuttke: 153-176. Bad Heilbrunn: Julius Klinkhardt.

Herzig, Bardo. 2012. Medienbildung. Grundlagen und Anwendungen. Handbuch Medienpädagogik Bd. I. München: kopaed.

Herzig, Bardo. 2014. «Wie wirksam sind digitale Medien im Unterricht?» Bielefeld: Bertelsmann Stiftung. http://www.digitalisierung-bildung.de/wp-content/uploads/2014/11/DigitaleMedienUnterricht_final.pdf.

Hromkovic, Juraj. 2012. "Ich staune über den informatischen Analphabetismus in der Schweiz». http://info.sonntagszeitung.ch/archiv/detail/?newsid=226954.

Humbert, Ludger. 2011. «Informatik, Informatische Bildung und Medienbildung». LOG IN 31(2011): 34-39

Jörissen, Benjamin und Winfried Marotzki. 2009. «Dimensionen Strukturaler Medienbildung». In Medienbildung - Eine Einführung, hrsg. v. Benjamin Jörissen und Winfried Marotzki, 30-40. Bad Heilbrunn: Julius Klinkhardt.

Keil, Reinhard. 2011. «Hypothesengeleitete Technikgestaltung als Grundlage einer kontextuellen Informatik.» In Informationstechnik und ihre Organisationslücken, hrsg. v. Andreas Breiter und Martin Wind: 165-184. Münster: LIT.

Kleiner, Paul. 2014. Was ist Informatik? Bern: Hasler Stiftung.

KMK - Kultusministerkonferenz. 1997. Neue Medien und Telekommunikation im Bildungswesen. Beschluss der Kultusministerkonferenz vom 28. Februar 1997. Berlin: Sekretariat der KMK.

KMK - Kultusministerkonferenz. 2016. Neue Medien und Telekommunikation im Bildungswesen. Bonn: KMK.

Knobelsdorf, Maria et al. 2015. «Computer Science Education in North-Rhine Westphalia, Germany - A Case Study». ACM Transactions on Computing Education 15(2015)2: Article No. 9.

Manovich, Lev. 2001. The Language of New Media. Cambridge, Massachusetts; London, England: The MIT Press.

Manovich, Lev. 2013. Software Takes Command. New York, London: Bloomsbury.

Moser, Heinz, Petra Grell, und Horst Niesyto, Hrsg. 2011. Medienbildung und Medienkompetenz. München: kopaed. http://www.medienpaed.com/issue/view/29.

Moser, Heinz. 2010. Einführung in die Medienpädagogik. Aufwachsen im Medienzeitalter. Wiesbaden: VS Verlag für Sozialwissenschaften.

Moser, Heinz. 2013. Kommentar zum Lehrplan 21. https://heinzmoser.wordpress. com/2013/07/22/lehrplan-21-es-droht-das-verschwinden-der-medienbildung/.

Patzlaff, Rainer. 2001. Der gefrorene Blick. Physiologische Wirkungen des Fernsehens und die Entwicklung des Kindes. Stuttgart: Verlag Freies Geistesleben.

Reiter, Anton. 2010. «Medienbildung auf der Überholspur. Ein Ersatz für die informatische Bildung?» In 25 Jahre Schulinformatik in Österreich. Zukunft mit Herkunft. Tagungsband (271), hrsg. v. Gerhard Brandhofer, 74-99. 
Schelhowe, Heidi. 2006. «Medienpädagogik und Informatik: Zur Notwendigkeit einer Neubestimmung der Rolle digitaler Medien in Bildungsprozessen». MedienPädagogik 12(22. März): 1-21. doi:10.21240/mpaed/12/2006.03.22.X.

Schelhowe, Heidi. 2007. «Interaktion und Interaktivität. Aufforderungen zu einer technologiebewussten Medienpädagogik». In Medienpädagogik - Standortbestimmung einer erziehungswissenschaftlichen Disziplin. Jahrbuch Medienpädagogik 6., hrsg. v. Werner Sesink, Michael Kerres und Heinz Moser, 144-160. Wiesbaden: VS Verlag für Sozialwissenschaften.

Schubert, Sigrid und Andreas Schwill. 2011. Didaktik der Informatik. 2. Auflage. Heidelberg: Spektrum Akademischer Verlag.

Senkbeil, Martin, Frank Goldhammer, Wilfried Bos, Birgit Eickelmann, Knut Schwippert, und Julia Gerick. 2014. «Das Konstrukt der computer- und informationsbezogenen Kompetenzen in ICILS 2013.» In ICILS 2013 - Computer- und informationsbezogene Kompetenzen von Schülerinnen und Schülern in der 8. Jahrgangsstufe im internationalen Vergleich, hrsg. v. Wilfried Bos, Birgit Eickelmann, Julia Gerick, Frank Goldhammer, Heike Schaumburg, Knut Schwippert, Martin Senkbeil, Renate Schulz-Zander und Heike Wendt, 83-112. Münster: Waxmann.

Sesink, Werner. 2009. «Zur bildungstheoretischen Bedeutung des Diskurses zwischen Pädagogik und Informatik». In Communication, Cooperation, Participation (CCP), Sonderausgabe Theorie der Informatik (9), 210-218.

Sesink, Werner. 2013. «Eine kritische Bildungstheorie der Medien». In Positionen der Medienbildung, hrsg. v. Winfried Marotzki und Norbert Meder, 127-158. Wiesbaden: VS Verlag für Sozialwissenschaften.

Spanhel, Dieter. 2006. Medienerziehung. München: kopaed.

Spannagel, Christian. 2015a. «Das große X. Informatik, informationstechnische Grundbildung, digitale Medienkompetenz?» L.A. multimedia (2): 30-33.

Spannagel, Christian. 2015b. Über das Verschwinden der Informatik in Baden-Württemberg. http://www.scilogs.de/bildungsluecke/ueber-verschwinden-informatik-baden-wuerttemberg/.

Steffen, Friedrich. 2003. «Informatik als Schulfach - wichtiger denn je!» LOG IN 23(2003): 10-14.

Tulodziecki, Gerhard, und Bardo Herzig. 2002. Computer \& Internet in Schule und Unterricht. Medienpädagogische Grundlagen und Beispiele. Berlin: Cornelsen Verlag Scriptor.

Tulodziecki, Gerhard, und Bardo Herzig. 2004. Mediendidaktik. Medien in Lehr- und Lernprozessen verwenden. München: kopaed.

Tulodziecki, Gerhard, Bardo Herzig, und Silke Grafe. 2010. Medienbildung in Schule und Unterricht. Bad Heilbrunn: Klinkhardt/UTB.

Wagner, Wolf-Rüdiger. 2013. Bildungsziel Medialitätsbewusstsein. Einladung zum Perspektivwechsel in der Medienbildung. München: kopaed.

Welling, Stefan, Ines Averbeck, und Julia Renke. 2013. «Evaluation des österreichischen Referenzmodells für digitale Kompetenzen». In Digitale Schule Österreich. Eine analoge Standortbestimmung anlässlich der eEducation Sommertagung 2013, 47-57, hrsg. v. Peter Micheuz, Anton Reiter, Gerhard Brandhofer et al. Österreichische Computer Gesellschaft. 
Wilkens, Ulrike. 2000. Das allmähliche Verschwinden der informationstechnischen Grundbildung. Zum Verhältnis von Informatik und Allgemeinbildung. Aachen: Shaker Verlag.

Wing, Jeannette M. 2006. «Computational Thinking». In Communication of the ACM. 49(3): 33-35.

Wing, Jeannette M. 2010. Computational Thinking: What and Why? https://www.cs.cmu. edu/ CompThink/resources/TheLinkWing.pdf.

Winkler, Hartmut. 2004. «Mediendefinition». Medienwissenschaft - Rezensionen, Reviews, Nr. 1/04, Mai 2004: 9-27.

Zorn, Isabel. 2011. «Medienkompetenz und Medienbildung mit Fokus auf Digitale Medien». In Medienbildung und Medienkompetenz, hrsg. v. Heinz Moser, Petra Grell und Horst Niesyto, 175-209. München: kopaed. doi:10.21240/mpaed/20/2011.09.19.X. 